\title{
De novo Evolution of a Small Choroidal Melanoma
}

\author{
Nino Aleksidze Carlos A. Medina Arun D. Singh \\ Department of Ophthalmic Oncology, Cole Eye Institute, Cleveland Clinic Foundation, \\ Cleveland, Ohio, USA
}

\section{Key Words}

De novo choroidal melanoma Choroidal neoplasms · Small choroidal melanoma Evolution

\begin{abstract}
Aim: To report the evolution of a de novo choroidal melanoma. Method: This is a case report of a 22-year-old white male patient who has been undergoing periodic examination for a choroidal 'freckle' since 10 years of age. Results: In 2007, a fundus photograph of the left eye showed a nondescript area of choroidal hyperpigmentation temporal to the fovea. Progressive growth was observed and, by 2012, the lesion had become well circumscribed and raised. One year later, a $4.5 \times 4.5 \times 1.5 \mathrm{~mm}$, dome-shaped, pigmented, choroidal mass with subretinal fluid and orange pigmentation was evident. The lesion was classified as a small choroidal melanoma. Six months after plaque radiotherapy, tumor regression with total resolution of the subretinal fluid was documented. Conclusion: The distinction between small choroidal melanoma and choroidal nevus is not absolute; therefore, some choroidal melanomas may initially be mislabeled as choroidal nevi because of their small size until continued growth identifies them to be small choroidal melanomas. In our case, the documented growth of the choroidal lesion on each consecutive visit and its high-risk features strongly suggest that it had been a melanoma from the beginning. To our knowledge, this is only the second documented case of a de novo evolution of a small choroidal melanoma.

(C) 2014 S. Karger AG, Basel
\end{abstract}


Ocular Oncology

and Pathology
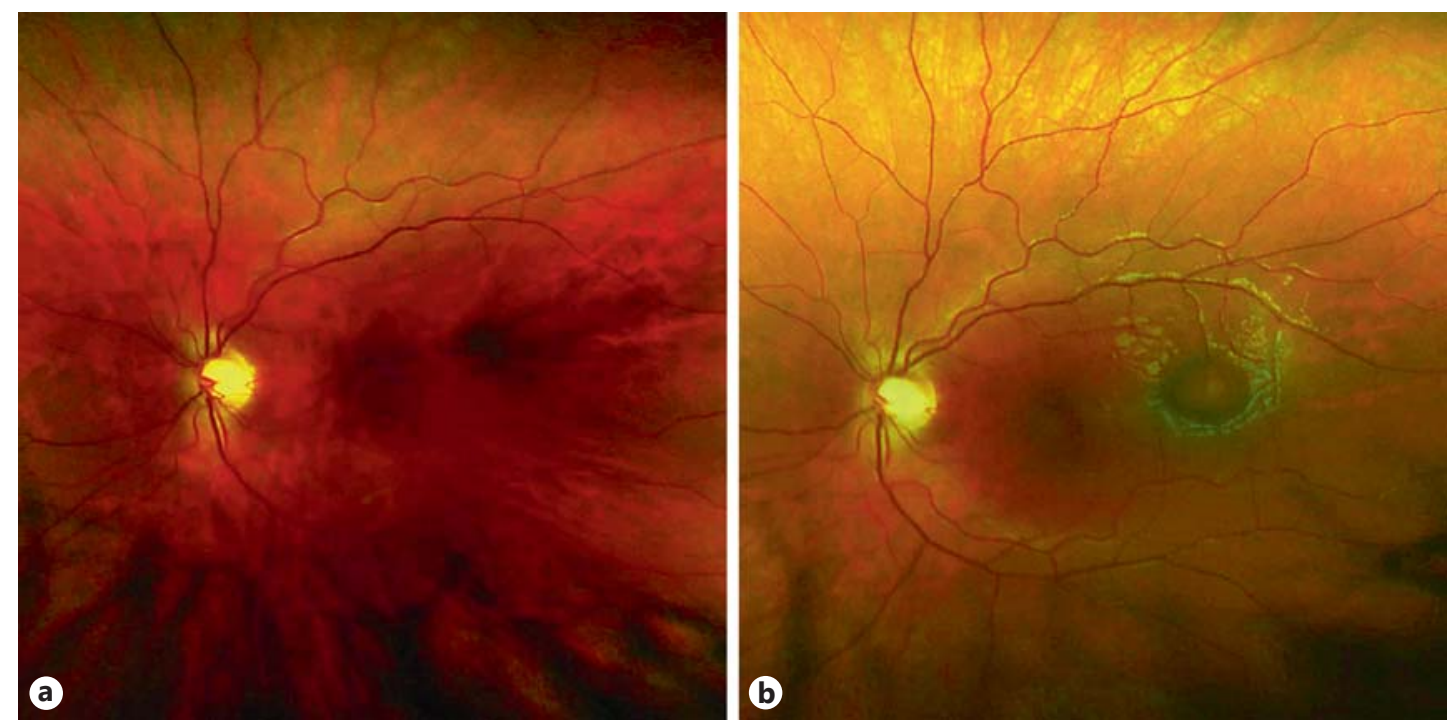

Fig. 1. a Fundus photograph of the left eye showing a nondescript area of choroidal hyperpigmentation temporal to the fovea. b Progressive growth was observed and, by 2012, the lesion was well circumscribed and raised.

\section{Introduction}

The estimated prevalence of choroidal nevi is fairly high, reaching $6 \%$ in the white population in the United States [1]. On the other hand, the incidence of uveal melanomas is rare, estimated to be 4.3 per million in the United States [2]. Although there is clinical and histopathological evidence suggesting that choroidal melanomas can arise from preexisting choroidal nevi $[3,4]$, choroidal melanomas may also arise de novo. To our knowledge, only one documented case of de novo choroidal melanoma development has been reported so far [5]. We herein report another case of a de novo development of a uveal melanoma in a young patient.

\section{Case Report}

A 22-year-old white male patient was referred for evaluation of a choroidal mass in his left eye. Prior to referral, he had been undergoing periodic examination for a choroidal lesion, diagnosed as a choroidal 'freckle' by his pediatric ophthalmologist at 10 years of age (in 2001). In 2007, the lesion was for the first time photographed when he was 15 years old (fig. 1a), followed by another photograph taken 5 years later at the age of 22 years (fig. 1b). The patient was monitored for another year before his referral to the Department of Ophthalmic Oncology at the Cole Eye Institute, Cleveland Clinic Foundation.

On examination, his visual acuity was 20/20 bilaterally. Anterior segment and slit lamp examinations were unremarkable in both eyes. Fundus examination of the right eye was unremarkable; however, on fundus examination of the left eye, a $4.5 \times 4.5 \times 1.5 \mathrm{~mm}$, dome-shaped, pigmented, choroidal mass with subretinal fluid and orange pigmentation was observed $2 \mathrm{~mm}$ temporal to the fovea (fig. 2a). The subretinal fluid did not extend into the fovea. The optic disc and macula were normal. Ultrasonography, OCT, fundus autofluorescence, fluorescein angiography and fundus photographs were taken to document the lesion (fig. $2 \mathrm{~b}-\mathrm{d}$ ). The presence of orange pigmentation was confirmed using autofluorescence, and OCT confirmed the presence of subretinal fluid. Previous fundus photographs from 2007 and 2012 were reviewed, and a progressive enlargement of the lesion was noted. 
Ocular Oncology

and Pathology
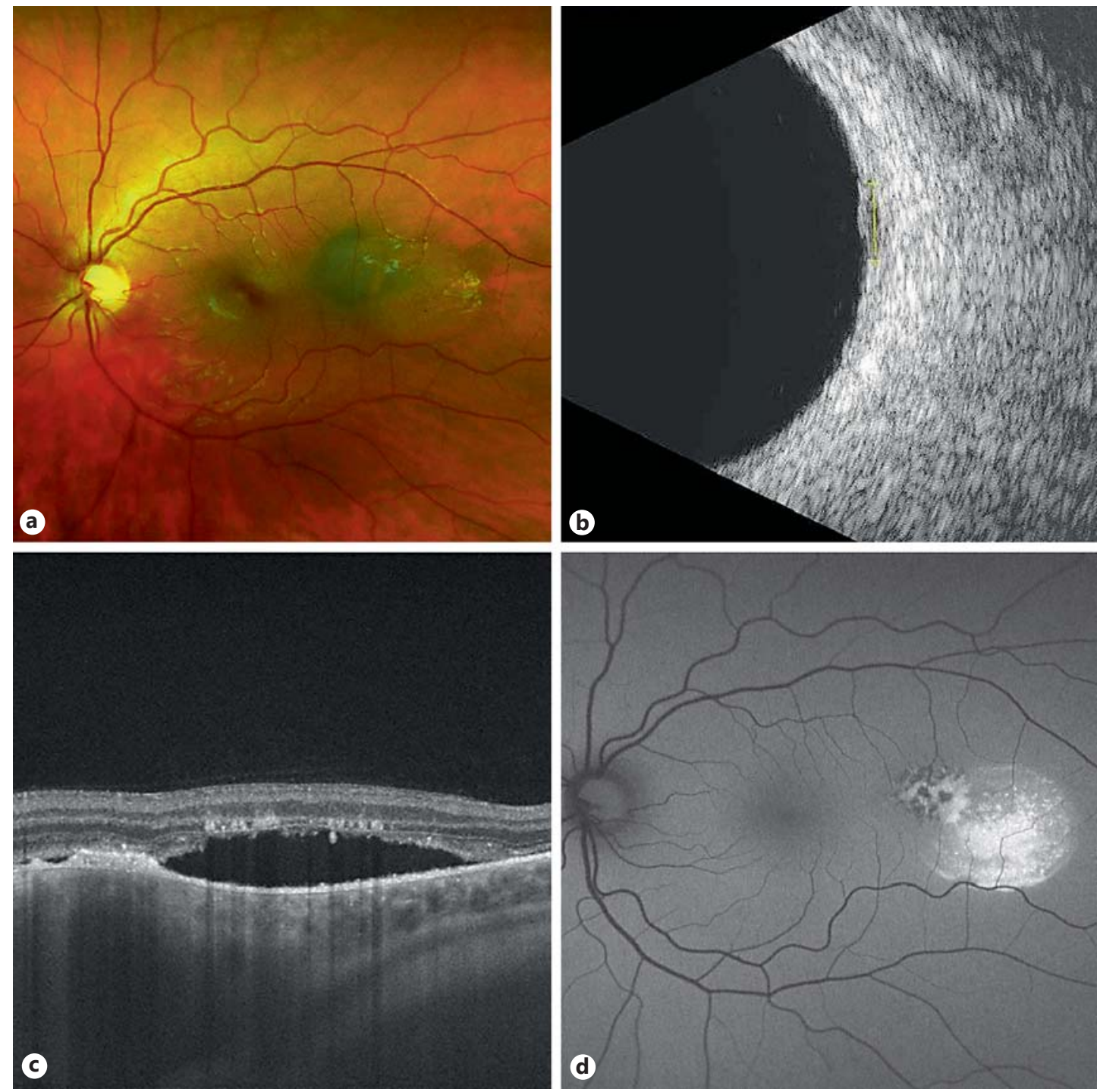

Fig. 2. Photographs taken at referral (2013). a A $4.5 \times 4.5 \times 1.5 \mathrm{~mm}$, dome-shaped, pigmented, choroidal mass with subretinal fluid and orange pigmentation temporal to the fovea is shown. $\mathbf{b}$ B-scan ultrasonography confirmed a dome-shaped mass. cSD-OCT over the lesion revealed subretinal fluid. The underlying choroidal lesion is not visualized. $\mathbf{d}$ Fundus autofluorescence showing hyperautofluorescence corresponding to the presence of lipofuscin.

The lesion was classified as a small choroidal melanoma according to criteria from the Collaborative Ocular Melanoma Study (COMS) [6]. The patient and his family were informed about the nature of uveal melanomas and about therapeutic options including continued observation, transpupillary thermotherapy, plaque radiotherapy and enucleation. Risks, benefits, alternatives and complications of each procedure were outlined. Following discussion, the patient chose to proceed with plaque radiotherapy (iodine-125, 85 Gy to the apex in $72 \mathrm{~h}$ ). Six months after surgery, tumor regression with total resolution of the subretinal fluid was evident (fig. 3).

Baseline systemic evaluation including CT scans of the chest, abdomen and pelvis was negative for systemic metastases. Subsequent systemic surveillance at 6 months after the initial diagnosis (hepatic panel and ultrasound of the liver) was also negative. 
Ocular Oncology

and Pathology
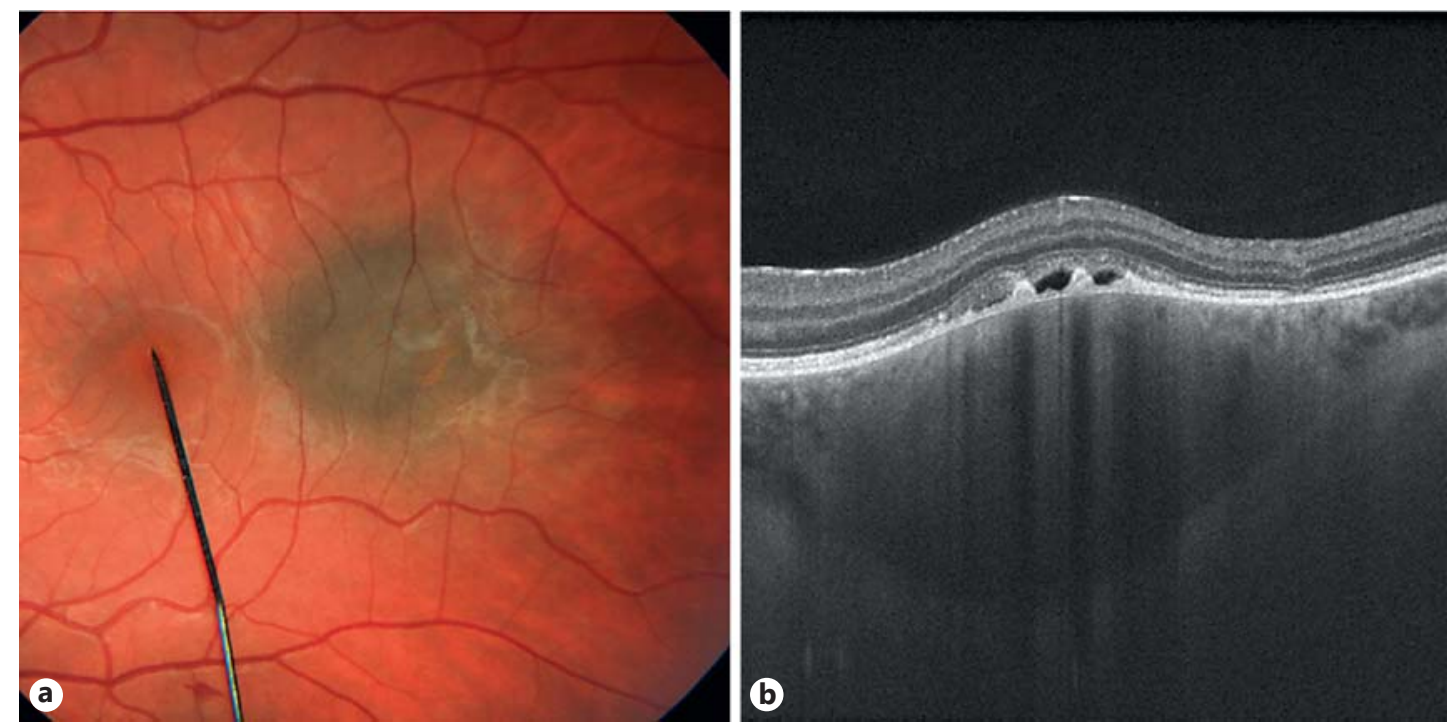

Fig. 3. Photographs taken 6 months after plaque radiation therapy in 2004. a Tumor regression of $4.5 \times 4.5$ $\times 1.1 \mathrm{~mm}$ can be observed. b OCT revealing resolution of subretinal fluid.

\section{Discussion}

Small pigmented choroidal lesions present a diagnostic and therapeutic dilemma to clinicians as the preferred method of treatment remains debatable [7]. There are several reasons for this controversy, but first and foremost there is a lack of any uniformly accepted terminology or diagnostic criteria of small choroidal melanoma $[8,9]$. The second issue is the lack of a randomized prospective trial to compare prompt treatment with deferred treatment until the diagnosis is confirmed (documented growth) [10].

A choroidal melanocytic lesion that is $<5 \mathrm{~mm}$ in base and $<1 \mathrm{~mm}$ in height is generally classified as a choroidal nevus. In a large cohort of patients, the risk of growth can be estimated according to the presence or absence of associated clinical features $[9,11,12]$. Nevertheless, in an individual patient, the distinction between small choroidal melanoma and choroidal nevus is not absolute $[9,11]$. This limitation exists because most of the risk factors predictive of growth, such as the presence of orange pigment and subretinal fluid, indicate secondary effects on adjacent tissues rather than the intrinsic composition of the lesion [9].

In a supplemental analysis of data from 188 small melanomas within the observational prospective study component of the COMS [11], a Kaplan-Meier estimate of the 5-year growth rate of $>50 \%$ was observed with the combination of two risk factors: height $(2.0-3.0 \mathrm{~mm})$ and diameter (12.1-16.0 mm), height (2.0-3.0 $\mathrm{mm}$ ) and presence of orange pigment, and diameter (12.1-16.0 $\mathrm{mm}$ ) and presence of orange pigment [13].

The published literature only refers to the risk of growth of choroidal nevi, which may include several biologic variants such as de novo choroidal melanomas that are initially mislabeled as choroidal nevi because of their size until continued growth identifies them to be small choroidal melanomas. The growth rate of a uveal melanoma is variable, with the median doubling times ranging from 154 to 511 days [14]. The other group comprises cases of longstanding choroidal nevi that undergo malignant transformation. The annual rate of malignant transformation of preexisting nevi is estimated to be 1 in 8,845, with an important assumption that all choroidal melanomas develop from preexisting nevi [1]. Given that some choroidal 
Aleksidze et al.: De novo Evolution of a Small Choroidal Melanoma

melanomas can arise from de novo, the true risk of malignant transformation of nevi into melanomas is expected to be lower and is hard to determine because of a lack of documentation of choroidal melanomas arising de novo.

The above-mentioned risk factors and the documented growth of the choroidal lesion on each consecutive visit strongly suggest that the choroidal lesion in our patient had been a melanoma (de novo) from its initial observation. To our knowledge, our patient is only the second documented case of a de novo evolution of a small choroidal melanoma.

\section{References}

1 Singh AD, Kalyani P, Topham A: Estimating the risk of malignant transformation of a choroidal nevus. Ophthalmology 2005;112:1784-1789.

-2 Singh AD, Turell ME, Topham AK: Uveal melanoma: trends in incidence, treatment, and survival. Ophthalmology 2011;118:1881-1885.

3 Yanoff M, Zimmerman LE: Histogenesis of malignant melanomas of the uvea. II. Relationship of uveal nevi to malignant melanomas. Cancer 1967;20:493-507.

4 Yanoff M, Zimmerman LE: Histogenesis of malignant melanomas of the uvea. III. The relationship of congenital ocular melanocytosis and neurofibromatosis in uveal melanomas. Arch Ophthalmol 1967;77:331-336.

5 Sahel JA, Pesavento R, Frederick AR Jr, Albert DM: Melanoma arising de novo over a 16-month period. Arch Ophthalmol 1988;106:381-385.

6 Group COMS: COMS Manual of Procedures (accession No. PBS 179693). Springfield, National Technical Information Service, 1995.

7 Gass JD: Problems in the differential diagnosis of choroidal nevi and malignant melanoma. XXXIII Edward Jackson Memorial lecture. Trans Sect Ophthalmol Am Acad Ophthalmol Otolaryngol 1977;83:19-48.

-8 Butler P, Char DH, Zarbin M, Kroll S: Natural history of indeterminate pigmented choroidal tumors. Ophthalmology 1994;101:710-716; discussion 717.

9 Singh AD, Mokashi AA, Bena JF, et al: Small choroidal melanocytic lesions: features predictive of growth. Ophthalmology 2006;113:1032-1039.

10 Kupfer C: Risk factors for growth and metastasis of small choroidal melanocytic lesions (discussion). Trans Am Ophthalmol Soc 1995;93:275-279.

11 Factors predictive of growth and treatment of small choroidal melanoma: COMS Report No. 5. The Collaborative Ocular Melanoma Study Group. Arch Ophthalmol 1997;115:1537-1544.

12 Shields CL, Furuta M, Berman EL, et al: Choroidal nevus transformation into melanoma: analysis of 2,514 consecutive cases. Arch Ophthalmol 2009;127:981-987.

13 Singh AD, Schachat AP, Diener-West M, Reynolds SM: Small choroidal melanoma. Ophthalmology 2008;115: 2319-2319e3.

14 Singh AD: Uveal melanoma: implications of tumor doubling time. Ophthalmology 2001;108:829-830. 